\title{
VIABILIDAD DE UNA PLATAFORMA DE E-COMMERCE PARA LA PRODUCCIÓN ECOLÓGICA EN LA COMUNIDAD VALENCIANA
}

\author{
Brugarolas, Margarita*; ; Martínez-Carrasco, Lauraa; Martínez-Poveda, África ${ }^{\text {a }}$;gulló-Torres, \\ Asunción ${ }^{a}$ \\ a Centro de Investigación e Innovación Agroalimentaria y Agroambiental (CIAGRO-UMH), Miguel \\ Hernandez University, Carretera de Beniel km 3.2, 03312 Orihuela, Alicante, Spain
}

\begin{abstract}
Resumen
El sector agrario valenciano, sustentado por empresas familiares con pequeñas producciones y una enorme diversidad de cultivos, representa un gran potencial para la explotación agroecológica. Sin embargo, el acceso a los mercados para los pequeños productores es complicado. En este escenario, se ha considerado la posibilidad de desarrollar una plataforma de venta online para productores ecológicos, que estaría gestionada por el organismo certificador del territorio (CAE-CV). El objetivo es determinar si los actores del mercado, productores y consumidores de productos ecológicos, estarían dispuestos a utilizar este tipo de comercio y establecer cuáles son las características más importantes que debe tener la herramienta. Se realizó una encuesta a consumidores y una entrevista a productores antes del desarrollo de la herramienta (2019). Con los resultados obtenidos se realizó una versión demo de la web, considerando los parámetros aportados por los actores y, finalmente, se evaluó la aceptación de la misma (2020). En los resultados, se observa una aceptación alta, tanto por parte de los consumidores como de los productores, y sobre todo un incremento importante de la intención de compra online por parte de los consumidores entre los períodos considerados, lo que muestra el creciente desarrollo de este tipo de canal.
\end{abstract}

Palabras claves: E-commerce, productos ecológicos, venta online, intención de compra

\section{Introducción}

La Unión Europea, en sus estrategias "De la granja a la mesa" y la "Estrategia de la UE sobre Biodiversidad para 2030", contempla un plan de acción para la agricultura ecológica entre cuyos objetivos está el conseguir que, en los próximos años, un $25 \%$ de las tierras de cultivo se destinen a la producción ecológica. En el marco del I Plan Valenciano de Producción Ecológica de la Comunitat Valenciana 2016-2020 se establecieron como retos el fomento de la producción y la comercialización ecológica y la promoción del conocimiento de la agroecología valenciana. La propuesta de una plataforma online común, que permita una oferta variada de productos ecológicos, puede facilitar a los pequeños productores el acceso a los mercados de proximidad de una forma más competitiva y eficiente y puede contribuir a alcanzar los retos planteados.

En el sector agroalimentario, el comercio online no ha sido demasiado utilizado hasta hace poco tiempo, estando las páginas web más centradas en incrementar la visibilidad de las empresas que en el comercio en sí (Granollers et al., 2013). En cualquier caso, Jin et al. (2017) determinaron que los consumidores, en su compra online, prefieren encontrar variedad de oferta y productos etiquetados como "verdes" o "ecológicos", con lo que podemos suponer que el comercio online puede ser una opción adecuada para los alimentos ecológicos. Existen pocas investigaciones centradas en la evaluación de páginas web en el ámbito agroalimentario. Algunos ejemplos son los trabajos de Davidson (2007), Carmona et al. (2014) o CristobalFransi et al. (2020), centrados en la evaluación por parte de los consumidores. En este caso, la evaluación de una página web que aúna a colectivos diversos, tiene unas características peculiares, ya que ésta, ha de ser realizada no solo por los compradores, sino también por los productores/elaboradores, que deberán dar de alta su explotación/empresa y gestionar las ventas de sus productos.

El objetivo del trabajo es determinar si los actores del mercado, productores y consumidores ecológicos, estarían dispuestos a utilizar este tipo de comercio y establecer cuáles son las características más importantes que debe tener la herramienta.

\section{Metodología}

El trabajo se desarrolla siguiendo el siguiente esquema (Gráfico 1). 
Gráfico 1. Esquema seguido para el desarrollo de una plataforma de comercio online para productos ecológicos

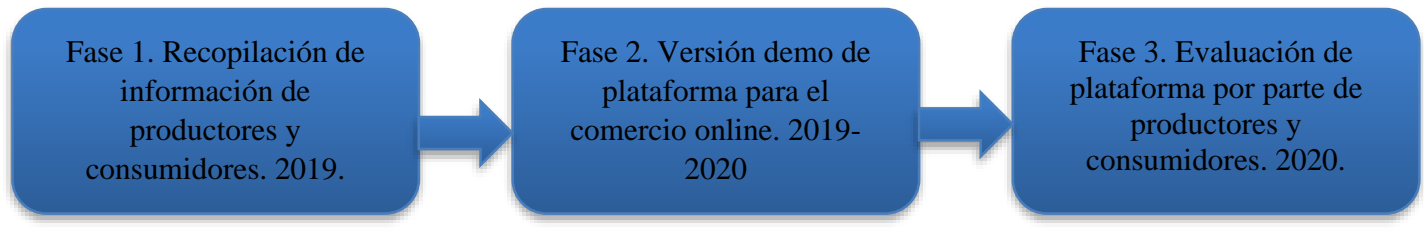

En la fase 1 se realizó una entrevista a productores ecológicos pertenecientes a la D.O. Granada Mollar de Elche y la D.O. Vinos de Alicante, ya que mostraron su interés en el proyecto. El número de entrevistas fue de 24 a productores ecológicos de granada (de una población de 44) y 9, a responsables de comercialización en bodegas de la D.O. Vinos de Alicante (de un total de 14). Se utilizó un cuestionario estructurado con tres bloques: i) preguntas generales sobre superficie y producción en ecológico y convencional, canales de distribución utilizados y mercados; ii) preguntas sobre la plataforma, como nivel de interés, ventajas e inconvenientes, y opciones concretas en caso de considerar su uso; iii) información personal del entrevistado. En esta fase se realizó también una encuesta online a consumidores ecológicos de la C.V con Google Formularios. Se obtuvieron 261 respuestas válidas. El cuestionario estaba estructurado en las siguientes secciones: i) hábitos de consumo de productos ecológicos; ii) hábitos de compra por internet; iii) aceptación de la plataforma y productos específicos que se comprarían a través de ella; iv) características específicas de la bioplataforma; v) características socioeconómicas de los encuestados.

Fase 2. Se desarrolló, con ayuda del Servicio de Apoyo Técnico a la Docencia y a la Investigación (SIATDI) de la UMH, una herramienta para el comercio electrónico de productos ecológicos que cumplía con los requerimientos expresados por productores y consumidores.

Fase 3. Se realizó la evaluación de la plataforma de comercio online. Para ello se realizaron dos encuestas virtuales, una a productores ecológicos y otra a consumidores. Se obtuvo una muestra de 86 productores ecológicos y 76 de consumidores ecológicos. En ambas se incluyó un enlace a la plataforma, unos enlaces a unos videotutoriales para su manejo en el caso de los productores, y un cuestionario en el que se evaluaron diferentes aspectos de la misma. Las encuestas incluyeron tres bloques de preguntas similares: i) preguntas generales sobre la explotación/hábitos de compra o consumo; ii) valoración de la página web; iii) características sociodemográficas.

En el Cuadro 1 se muestran los factores incluidos en la valoración, definidos por diferentes ítems que fueron valorados en una escala de 1 a 5, así como el colectivo que los evaluó y los antecedentes bibliográficos que ayudaron a definir los ítems.

Cuadro 1. Antecedentes bibliográficos utilizados para la definición de los ítems incluidos en la valoración de la plataforma

\begin{tabular}{|c|c|c|}
\hline Ítems sobre... & Encuesta & Referencias \\
\hline $\begin{array}{l}\text { Contenido de la } \\
\text { web }\end{array}$ & $\begin{array}{l}\text { Productores y } \\
\text { consumidores }\end{array}$ & $\begin{array}{l}\text { Kong \& Liu, 2005; Parasuraman et al., 2005; Yi-wen et al., 2007; } \\
\text { Kaya, 2010; Ağı̈rgün, B. (2012); Ryan et al., (2012); Vatansever, } \\
\& \text { Akgul, 2014; Alptekin et al., 2015; Zaim et al., 2016; Liang et } \\
\text { al., 2017; Sun et al., (2017) }\end{array}$ \\
\hline $\begin{array}{l}\text { Calidad del } \\
\text { sistema de la web }\end{array}$ & $\begin{array}{l}\text { Productores y } \\
\text { consumidores }\end{array}$ & $\begin{array}{l}\text { Parasuraman et al., 2005; Kong \& liu, 2005; Yi-wen et al., 2007; } \\
\text { Kaya, 2010; Yu et al., 2011; Ağırgün, 2012; Aydin \& Kahraman, } \\
\text { 2012; El-Sofany et al, 2012; Ling \& Salvendi, 2013; Vatansever } \\
\text { \& Akgul, 2014; Alptekin et al., 2015; Liang et al, } 2017\end{array}$ \\
\hline $\begin{array}{l}\text { Valoración global } \\
\text { de la plataforma }\end{array}$ & $\begin{array}{l}\text { Productores y } \\
\text { consumidores }\end{array}$ & Parasuraman et al., 2005 \\
\hline $\begin{array}{l}\text { Calidad del } \\
\text { servicio de la web }\end{array}$ & Consumidores & $\begin{array}{l}\text { Ağırgün, 2012; Aydin \& Kahraman, 2012; El Sofany et al., } \\
\text { 2012; Vatansever, \& Akgul, 2014; Alptekin et al., 2015; Liang } \\
\text { et al, } 2017\end{array}$ \\
\hline $\begin{array}{l}\text { Actividad propia } \\
\text { en la web }\end{array}$ & Productores & Alptekin et al., 2015; Liang et al, 2017 \\
\hline $\begin{array}{l}\text { Información propia } \\
\text { en la web }\end{array}$ & Productores & Encuesta de consumidores del periodo anterior \\
\hline
\end{tabular}




\section{Resultados}

Se mostrarán en este apartado los resultados obtenidos en la fase 3, ya que en la fase 1, el interés mostrado por los dos grupos de actores fue elevado y permitió la continuidad del proyecto. Los requerimientos que indicaron ambos colectivos fueron incorporados en la versión a evaluar en esta fase.

\subsection{Resultados de los productores ecológicos}

La valoración global de la web por parte de los productores es bastante positiva, ya que recomendarían la web a otros productores $(3,96 / 5)$ y consideran la utilización de la web una experiencia agradable $(3,93 / 5)$. Sin embargo, en cuanto a la utilización de la web para la venta de los productos, la puntuación, aunque positiva, no es demasiado alta $(3,46 / 5)$. En este caso se observa que un $47,3 \%$ de los respondientes se muestra dispuesto a utilizarla, aunque hay un porcentaje alto de productores que están indecisos $(41,9 \%)$ y un $10,5 \%$ de los productores indicaron que probablemente no la utilizarían.

En cuanto a las características de la plataforma, consideran que el contenido y el servicio que ofrece la web son claros $(3,83 / 5)$ y consideran que la oferta de otros productos de competidores no solo es positiva $(4,10 / 5)$, sino que puede favorecer la venta de sus productos $(3,86 / 5)$. Entre los aspectos considerados para evaluar la calidad del sistema de la web destaca el diseño atractivo (4,05/5), la sencillez de navegación (3,96/5), la rapidez de carga de las imágenes (3,95/5), la rapidez de navegación por la web y entre páginas $(3,94 / 5)$ y relación entre las imágenes y el contenido que muestran $(3,88 / 5)$. En lo que respecta a las acciones que deben realizar para comercializar sus productos y la información que mostrarán en la web, como la sencillez del proceso, el alta como productor, la incorporación de productos, las nuevas ofertas, el aviso de nuevos pedidos, la ubicación en el mapa de la explotación o los canales de comunicación con los clientes, las puntuaciones también son favorables, aunque tienen un cierto margen de mejora (3,7 a 3,49/5).

\subsection{Resultados de los consumidores}

Los consumidores se muestran algo más dispuestos que los productores a utilizar la web para comprar alimentos (3,54/5). El porcentaje de los que utilizaría la plataforma es de un $61,0 \%$, el de indecisos de un $27,1 \%$ y el de los que no la usarían de un $11,9 \%$. Además, el porcentaje de consumidores que compran alimentos online se ha incrementado respecto a la primera fase, posiblemente debido a la pandemia.

Las puntuaciones para los ítems relacionados con el contenido de la web son altos. Destacan aspectos como la información sobre los productores $(3,97 / 5)$ o la ubicación de las explotaciones $(3,95 / 5)$, los idiomas que se utilizan $(3,9 / 5)$, los métodos de pago ofrecidos $(3,87 / 5)$ y la claridad de los contenidos y servicios $(3,85 / 5)$. Otros aspectos valorados positivamente, aunque con margen de mejora son: el interés personal en los productos ofrecidos $(3,66 / 5)$, la información sobre los productos $(3,62 / 5)$, la actualización de los productos $(3,32 / 5)$ y la información incluida en la página de descripción de cada producto $(3,35 / 5)$. Tan solo la gama de productos ofrecida les parece incompleta $(2,85 / 5)$. Esto puede ser debido a que, al tratarse de una versión de prueba, se mostraban solo unos pocos productos, pero si los productores deciden utilizar la plataforma, la gama se ampliará notablemente. La calidad del sistema de la web también ha recibido valoración positiva de los consumidores, sobre todo en lo que respecta a la rapidez con la que se cargan las imágenes $(4,02 / 5)$ y el diseño atractivo $(4,0 / 5)$. Les parece rápido y sencillo navegar por la web y entre páginas ( $3,95 / 5$ y $3,90 / 5$, respectivamente). También consideran que los textos utilizados en los enlaces describen satisfactoriamente lo que se ofrece en las páginas a las que dan acceso $(3,85 / 5)$, así como lo adecuado de las imágenes para mostrar el contenido $(3,76 / 5)$. Con puntuaciones algo menores tenemos, el que las búsquedas dirigen convenientemente al producto buscado $(3,66 / 5)$, la evaluación sobre la adecuación del producto que pueden realizar en la página de resultados de las búsquedas $(3,55 / 5)$, la facilidad para ordenar y filtrar $(3,53 / 5)$ y el que tenga todos los filtros necesarios $(3,35 / 5)$. En cuanto a la valoración de la calidad del servicio de la web destaca la confianza en los productos ofertados $(4,0 / 5)$. Consideran que pueden valorar si un producto es serio y confiable fácilmente $(3,79 / 5)$ y su experiencia de compra $(3,79 / 5)$. Con menor puntuación destacan la facilidad para hacer sugerencias y para contactar con los productores $(3,56 / 5)$.

\section{Conclusiones}

La plataforma se plantea como una alternativa de comercialización para los productores ecológicos que tendrán un acceso más directo a los consumidores. La aceptación de la misma por parte de los consumidores es elevada, sin embargo, la disposición a usarla de los productores es algo menor, lo que puede denotar su reticencia a la adopción de nuevas tecnologías. Si no se consigue un volumen amplio de productores implicados y con una oferta variada, el interés de los consumidores podría disminuir, por lo que es importante que el colectivo de productores se implique. Parece importante apuntar que para el manejo de la plataforma será necesaria una organización y planificación externa, que posiblemente pudiera estar vinculada al CAE-CV, como organismo de garantía, y convendría realizar programas de formación ad hoc para los productores. 


\section{Bibliografía}

Carmona, C. J., Ramírez-Gallego, S., Torres, F., Bernal, E., del Jesus, M. J. y García, S. (2012). Web usage mining to improve the design of an e-commerce website: OrOliveSur. com. Expert Systems with Applications, 39(12): 11243-11249.

Cristobal-Fransi, E., Montegut-Salla, Y., Ferrer-Rosell, B. y Daries, N. (2020). Rural cooperatives in the digital age: An analysis of the Internet presence and degree of maturity of agri-food cooperatives'ecommerce. Journal of Rural Studies, 74: 55-66.

Davidson, R. (2007). Five year longitudinal study of Australian winery websites. The 13th Asia Pacific Management Conference. Adelaida. Visto en: https://digital.library.adelaide.edu.au/dspace/bitstream/2440/55524/1/hdl_55524.pdf. Acceso [15 de octubre de 2020].

Granollers Saltiveri, T., Cristóbal Fransi, E. y Montegut Salla, Y. (2013). Análisis de usabilidad de cooperativas del sector de la fruta y aceite en el área de Lleida. RISTI: Iberian Journal on Information Systems \& Technologies/Revista Ibérica de Sistemas e Tecnologias de Informação, 11(6): 45-59.

Jin, S., Li, H. y Li, Y. (2017). Preferences of Chinese consumers for the attributes of fresh produce portfolios in an e-commerce environment. British Food Journal, 119(4): 817-829.

Ağırgün, B. (2012). Ranking B2C web sites with AHP and TOPSIS under fuzzy environment. Nevşehir Bilim ve Teknoloji Dergisi, 1(2): 65-78.

Alptekin, N., Hall, E. E. y Sevim, N. (2015). Evaluation of websites quality using fuzzy TOPSIS method. International Journal of Academic Research in Business and Social Sciences, 5(8): 221-242.

Aydin, S. y Kahraman, C. (2012). Evaluation of e-commerce website quality using fuzzy multi-criteria decision making approach. IAENG International Journal of Computer Science, 39(1): 64-70.

El-Sofany, H., Al-Malki, T., Alzamel, A. A.y Alharbi, A. A. (2012). Impact of Trust Factors in Improvement and Development of E-commerce in Saudi Arabia. International Journal of Computer Applications, 55(9): 15-24.

Kaya, T. (2010). Multi-attribute evaluation of website quality in E-business using an integrated fuzzy AHPTOPSIS methodology. International Journal of Computational Intelligence Systems, 3(3): 301314.

Kong, F. y Liu, H. (2005). Applying fuzzy analytic hierarchy process to evaluate success factors of ecommerce. International Journal of Information and Systems Sciences, 1(3-4): 406-412.

Liang, R., Wang, J. y Zhang, H. (2017). Evaluation of e-commerce websites: An integrated approach under a single-valued trapezoidal neutrosophic environment. Knowledge-Based Systems, 135: 44-59.

Ling, C., y Salvendy, G. (2013). Prioritising usability considerations on B2C websites. Theoretical Issues in Ergonomics Science, 14(1): 69-98.

Parasuraman, A., Zeithaml, V. A. y Malhotra, A. (2005). ES-QUAL: A multiple-item scale for assessing electronic service quality. Journal of service research, 7(3): 213-233.

Ryan, J. K., Sun, D. y Zhao, X. (2012). Competition and coordination in online marketplaces. Production and Operations Management, 21(6): 997-1014.

Sun, C., Ji, Y., Kolfal, B. y Patterson, R. (2017). Business-to-Consumer Platform Strategy: How Vendor Certification Changes Platform and Seller Incentives. ACM Transactions on Management Information Systems (TMIS), 8(2-3): 1-42.

Vatansever, K. y Akgul, Y. (2014). Applying fuzzy analytic hierarchy process for evaluating service quality of private shopping website quality: A case study in Turkey. Journal of Business Economics and Finance, 3(3): 283-301.

Yi-wen, L., Kwon, Y. y Kang, B. (2007). A fuzzy AHP approach to evaluating e-commerce websites. In Fifth International Conference on Software Engineering Research, Management and Applications (pp. 114-122). IEEE. https://doi.org/10.1109/SERA.2007.29

Yu, X., Guo, S., Guo, J. y Huang, X. (2011). Rank B2C e-commerce websites in e-alliance based on AHP and fuzzy TOPSIS. Expert Systems with Applications, 38(4): 3550-3557.

Zaim, H., Ramdani, M. y Haddi, A. (2016, October). Multi-criteria analysis approach based on consumer satisfaction to rank B2C E-commerce websites. In 2016 11th International Conference on Intelligent Systems: Theories and Applications (SITA) (pp. 1-6). IEEE. 